\title{
Nature-Inspired Planner Agent for Health Care
}

\author{
Javier Bajo $^{1}$, Dante I. Tapia ${ }^{2}$, Sara Rodríguez ${ }^{2}$, Ana de Luis ${ }^{2}$, and Juan M. Corchado ${ }^{2}$ \\ ${ }^{1}$ Universidad Pontificia de Salamanca, \\ Compañía 5, 37002, Salamanca, Spain \\ jbajope@upsa.es \\ ${ }^{2}$ Departamento Informática y Automática, Universidad de Salamanca \\ Plaza de la Merced s/n 37008, Salamanca, Spain \\ \{dantetapia, srg, adeluis, corchado\}@usal.es
}

\begin{abstract}
This paper presents an autonomous intelligent agent with a human thinking reasoning model, based on past experiences. The agent is developed to assist medical staff in geriatric residences. The health care process is a vital function, requiring nature-inspired solutions imitating the residence staff behaviours. An autonomous deliberative Case-Based Planner agent, AGALZ (Autonomous aGent for monitoring ALZheimer patients), is developed and integrated into an environment-aware multi-agent system, named ALZ-MAS (ALZheimer Multi-Agent System), to optimize health care in geriatric residences. ALZ-MAS is capable of obtaining information about the environment through RFID technology.
\end{abstract}

\section{Introduction}

Agents and multi-agent systems (MAS) have become increasingly relevant for developing distributed and dynamic intelligent environments. The ability of software agents to act somewhat autonomously links them with living animals and humans, so they seem appropriate for discussion under nature-inspired computing [7]. This paper presents AGALZ (Autonomous aGent for monitoring ALZheimer patients), and explains how this deliberative planning agent has been designed and implemented. A case study is then presented, with AGALZ working with complementary agents into a prototype environment-aware multi-agent system (ALZ-MAS: ALZheimer MultiAgent System). The elderly health care problem is studied, and the possibilities of Radio Frequency Identification (RFID) [10] as a technology for constructing an intelligent environment and ascertaining patient location to generate plans and maximize safety are examined.

This paper focuses in the development of nature-inspired deliberative agents using a Case-Based Reasoning (CBR) [1] architecture, as a way to implement sensitive and adaptive systems to improve assistance and health care support for elderly and people with disabilities, in particular with Alzheimer. Agents in this context must be able to respond to events, take the initiative according to their goals, communicate with other agents, interact with users, and make use of past experiences to find the best plans to achieve goals, so we propose the development of an autonomous deliberative agent that incorporates a Case-Based Planning (CBP) mechanism, derivative from CaseBased Reasoning (CBR) [2], specially designed for planning construction. CBP-BDI 
facilitates learning and adaptation, and therefore a greater degree of autonomy than that found in pure BDI (Believe, Desire, Intention) architecture [3]. BDI agents can be implemented by using different tools, such as Jadex [9], dealing with the concepts of beliefs, goals and plans, as java objects that can be created and handled within the agent at execution time.

During the last three decades the number of Europeans over 60 years old has risen by about $50 \%$. Today they represent more than $25 \%$ of the population and it is estimated that in 20 years this percentage will rise to one third of the population, meaning 100 millions of citizens [4]. This situation is not exclusive to Europe, since studies in other parts of the world show similar tendencies [4]. The importance of developing new and more reliable ways to provide care and support to the elderly is underlined by this trend [4], and the creation of secure, unobtrusive and adaptable environments for monitoring and optimizing health care will become vital. Some authors [8] consider that tomorrow's health care institutions will be equipped with intelligent systems capable of interacting with humans. Multi-agent systems and architectures based on intelligent devices have recently been explored as supervision systems for medical care for the elderly or Alzheimer patients, aimed to support them in all aspects of daily life, predicting potential hazardous situations and delivering physical and cognitive support.

RFID technology is a wireless technology used to identify and receive information on the move. An RFID system contains basically four components: tags, readers, antennas and software [10]. The configuration used in the system presented in this paper consists of $125 \mathrm{KHZ}$ transponders mounted on bracelets worn on the patient's wrist or ankle, several readers installed over protected zones, with up to 2 meters capture range, and a central computer where all the ID numbers sent by the readers is processed.

In the next section the autonomous nature-inspired health care agent, named AGALZ, is presented. Then, a case study is presented, describing the main characteristics of ALZ-MAS architecture and its agents, including AGALZ, finalizing with initial results and conclusions obtained after the implementation of a prototype into a real scenario.

\section{Autonomous Nature-Inspired Health Care Agent}

We have developed AGALZ, an autonomous deliberative Cased-Based Planner (CBPBDI) agent that integrates with other agents into a multi-agent system, named ALZMAS, as a proposal to improve the efficiency of health care and supervision of patients in geriatric residences. AGALZ presents a deliberative architecture, based on the BDI (Belief, Desire, Intention) model [3]. In this model, the internal structure and capabilities of the agents are based on human mental aptitudes, using beliefs, desires, and intentions. Our method facilitates the incorporation of CBR systems [1] as a deliberative mechanism within BDI agents, facilitating learning and adaptation and providing a greater degree of autonomy than pure BDI architecture. A deliberative CBP-BDI agent is specialized in generating plans and incorporates a Case-Based Planning (CBP) mechanism. The purpose of a CBR agents is to solve new problems by adapting solutions that have been used to solve similar problems in the past [1], and the CBP agents are a variation of the CBR agents, based on the plans generated from each case. A CBP planner is used for AGALZ to find plans to give daily nursing care in a 
geriatric residence $[2,6]$. It is very important maintaining a map with the location of the different elements that take part in the system at the moment of planning or replanning, so using RFID technology facilitates enormously the dynamic planning.

CBR is a type of human thinking based on reasoning about past experiences. To introduce a CBR motor into a BDI agent it is necessary to represent the cases used in a CBR system by means of beliefs, desires and intentions, and implement a CBR cycle. A case is a past experience composed of three elements: an initial state or problem description that is represented as a belief; a final state that is represented as a set of goals and a solution (belief); and the sequence of actions that makes it possible to evolve from an initial state to a final state. This sequence of actions is represented as intentions or plans. Figure 1 shows the internal structure of a CPB-BDI agent.

In a planner agent, the reasoning motor generates plans using past experiences and planning strategies, so the concept of Case-Based Planning is obtained [5, 6]. CBP consists of four sequential stages: retrieve stage to recover the most similar past experiences to the current one; reuse stage to combine the retrieved solutions in order to obtain a new optimal solution; revise stage to evaluate the obtained solution; and retain stage to learn from the new experience.

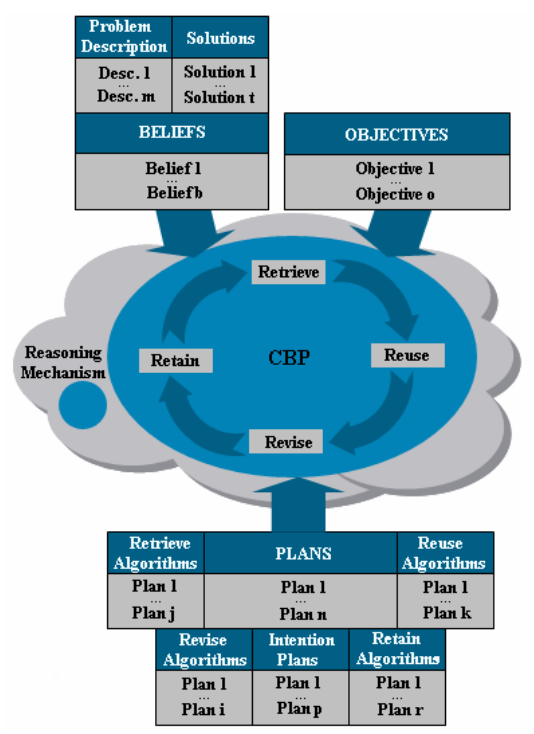

Fig. 1. CBP-BDI Agent internal structure

The CBP cycle is implemented through goals and plans. When the goal corresponding to one of the stages is triggered, different plans (algorithms) can be executed concurrently to achieve the goal. Each plan can trigger new sub-goals and, consequently, cause the execution of new plans. Deliberative CBP-BDI agents, like AGALZ, are able to incorporate other reasoning mechanisms that can coexist with the CBP. AGALZ is an autonomous agent that can survive in dynamic environments. However, is possible to incorporate communication mechanisms that allow it to be easily integrated into a multi-agent system and work coordinately with other agents to solve problems in a distributed way. 
The CBP planner constructs plans in such a way that a plan is a sequence of tasks that need to be carried out by a nurse. A task is a java object that contains the date of the requested service, the description of the service and the time limits to carry it out, as can be seen in Table 1 .

Table 1. Time (minutes) spent on indirect tasks

\begin{tabular}{ll}
\hline Task & Data \\
\hline TaskId & 36 \\
TaskType & 32 \\
TaskDescript & Feeding (lunch) \\
TaskPriority & 3 \\
TaskObjective & 0 \\
TaskIncidents & 0 \\
PatientId & 7 \\
PatientDependence & 2 \\
MinTime & $12: 30$ \\
MaxTime & $15: 00$ \\
TaskResources & Food \\
\hline
\end{tabular}

For each task one or more goals are established, in such a way that that the whole task is eventually achieved. A problem description will be formed by the tasks that the nurse needs to execute, the resources available, and the times assigned for their shift. In the retrieve stage, those problem descriptions found within a range of similarity close to the original problem description are recovered from the beliefs base. In our case, a tolerance of $20 \%$ has been permitted. In order to do this, AGALZ allows the application of different similarity algorithms (cosine, clustering etc.). Once the most similar problem descriptions have been selected, the solutions associated with them are recovered. One solution contains all the plans (sequences of tasks) carried out in order to achieve the objectives of AGALZ for a problem description (assuming that replanning is possible) in the past, as well as the efficiency of the solution being supplied. The chosen solutions are combined in the reuse stage to construct a plan [2,6]. The reuse is focused on the objectives and resources needed by each task, as well as on the objectives that the nurse needs to perform and the resources available in order to carry out the global plan. The objectives that each nurse has are aimed to attend the patients and not exceed eight nurse' working hours. The time available is a problem restriction. The resources necessary for some of the tasks are food, equipment and rooms, among others. AGALZ takes care of incidents and interruptions that may occur during replanning [2]. Furthermore AGALZ trusts the nurse in the sense that the revision of a plan is made by the nurse. Finally, AGALZ learns about this new experience. If the evaluation of the plan is at least a $90 \%$ similar, the case is stored in the cases memory.

\section{Case Study}

The Alzheimer Santisima Trinidad (ST) Residence of Salamanca has been interested in improving the services offered to its patients and has collaborated in the development of the technology presented here, providing their know-how and experimenting with the prototype developed. This residence is intended for people over 65 years old, and has the following services and facilities, among others: TV 


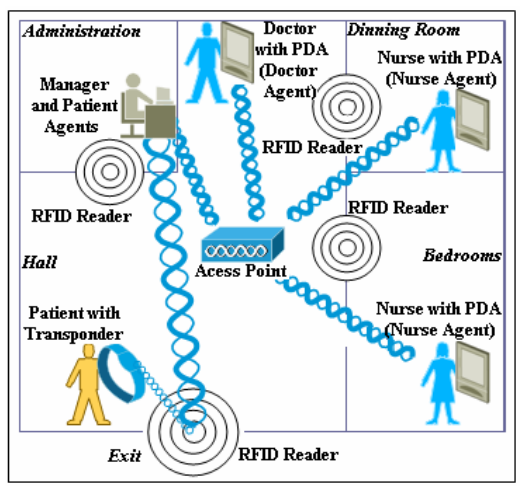

Fig. 2. ALZ-MAS wireless technology organization schema

room, geriatric bathroom, hairdressing salon, medical service, religious attention, occupational therapy, technical assistance, terrace, garden, laundry service, clothes adjustment, infirmary, reading room, living room, room of visits, cafeteria, social worker, chapel, elevator, customized diet, and multipurpose room.

Figure 2 shows a basic schema of the technology implemented in the residence. This residence has capacity for 60 patients, an average of 6 nurses, one social worker and 5 more employees with other responsibilities. We selected 30 patients to test the system, so the hardware implemented at the residence basically consisted of 42 ID door readers, one on each door and elevator, 4 controllers, one at each exit, one in the first floor hall and another in the second floor hall, and 36 bracelets, one for each patient and the nurses. The ID door readers get the ID number from the bracelets and send the data to the controllers which send a notification to the Manager agent, located in a central computer. To test the system 30 Patient agents, 10 AGALZ agents, 2 Doctor agents and 1 Manager agent were instantiated.

\subsection{ALZ-MAS: Alzheimer Health Care Multi-agent System}

The characteristics of multi-agent systems make them appropriate for implementing into geriatric residences to improve health care of the patients [8]. A multi-agent

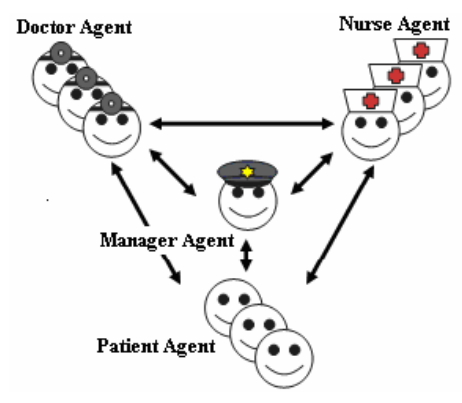

Fig. 3. ALZ-MAS architecture: Doctor, AGALZ, Patient and Manager agents, within their interactions 
system is a distributed system based on the cooperation of autonomous agents. The relationships established between the agents of ALZ-MAS are inspired in human's behaviours (doctors, nurses, patients, security guards, etc.) [7].

The conclusions obtained after studying the requirements of the problem are that ALZ-MAS is composed of four different agent types as shown in Figure 3:

- Patient agent manages the patient's personal data and behaviour (monitoring, location, daily tasks, and anomalies). Every hour validates the patient location, monitors the patient state and sends a copy of its memory base (patient state, goals and plans) to the manager agent in order to maintain backups. The patient state is instantiated at execution time as a set of beliefs and these beliefs are controlled through goals that must be achieved or maintained. The beliefs that were seen to define a general patient state at the Residence, were: weight, temperature, blood pressure, feeding (diet characteristics and next time to eat), oral medication, parenteral medication, posture change, toileting, personal hygiene, and exercise. The beliefs and goals used for every patient depend on the plan (treatment) or plans that the doctors prescribe. The patient agent monitors the patient state by means of the goals. To know if a goal has been achieved or has failed, it is necessary to maintain continuous communication with the rest of the ALZ-MAS agents, especially with AGALZ (through which the nurse can communicate the result of her assigned tasks). At least once per day, depending on the corresponding treatment, the patient agent must contact the nurse agent. The patient agent must have periodic communication with the doctor agent. Finally the patient agent must ensure that all the actions indicated in the treatment are taken out. Patient agents run on a central computer.

- Manager agent plays two roles the security role that controls the patients' location and manages locks and alarms; and the Manager role that manages the medical record database and the doctor-patient and nurse-patient assignment. It must provide security for the patients and medical staff and the patients, doctors and nurse assignment must be efficient. This assignation is carried out through a CBR reasoning engine, which is incorporated within the Manager agent. When a new assignation of tasks needs to be carried out to the nurses or from the patients to the doctors, both past experiences, such as the profile of the nurse or doctor, and the needs of the current situation are recalled. In this way tasks are allocated to the nurses. A nurse profile includes nurse's preferences such as hollidays, etc. Manager agent runs on a central computer.

- Doctor agent treats patients. It needs to interact with Patient agents to order treatments and receive periodic reports, with the Manager agent to consult medical records and assigned patients, and with AGALZ agent to ascertain patients' evolution.

- AGALZ schedules the nurse's working day obtaining dynamic plans depending on the tasks needed for each assigned patient. AGALZ manages nurses' profiles, tasks, available time and resources. The generated plans must guarantee that all the patients assigned to the nurse are given care. The nurse can't exceed 8 working hours. Every agent generates personalized plans depending on the nurse's profile and working habits. AGALZ agents run on mobile devices, where each nurse can see her plans task by task. A plan can be 
interrupted for different reasons: a resource fails, a patient suffers some sort of crisis and requires unforeseen attention, a patient has an unexpected visit or visits to a patient gone on over the permitted time allowed, etc.

\section{Results and Conclusions}

Figure 4 shows the average number of nurses working simultaneously (each of the 24 hours of the day) at the Residence before and after the implantation of the system prototype, with data collected from October 2005 to March 2006. The prototype was adopted on January $15^{\text {th }}, 2006$. The average number of patients was the same before and after the implementation. The tasks executed by nurses were divided in two categories: direct action tasks (where the nurses are in contact with the patients) and indirect action tasks (where nurses are not directly involved with patients, like monitoring, written reports, managing personal visits to the patients, etc.). During the first period the problem was analysed, the residence was observed and data was retrieved. Finally averages of the time spent by nurses in the carrying out of the tasks for every patient were obtained, having into account that a task depends on the dependency level of a patient and the nurse skill. For the direct action tasks, the following times were obtained for each patient: 35' cleaning, 18' feeding, 8' oral medication, 30' parenteral medication, 25' posture change, 8' toileting, 60' exercise and 10' others. We are especially interested on time spent on indirect action tasks; daily average times obtained for every kind of task before and after the implementation for each task can be seen on Table 2 .

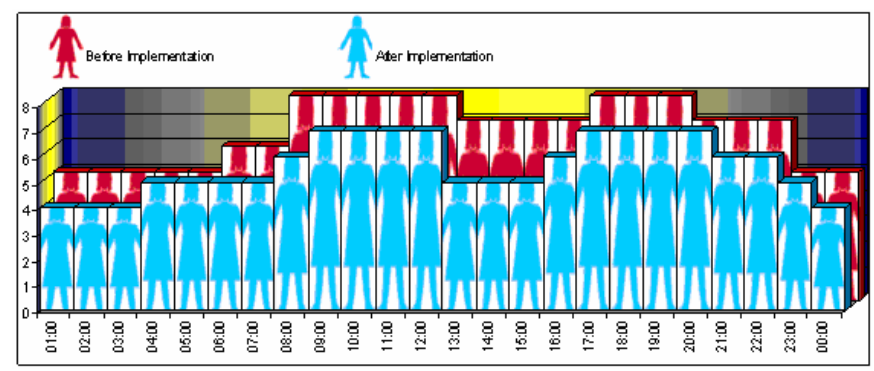

Fig. 4. Number of nurses working simultaneously in the residence

The system facilitates the more flexible assignation of the working shifts at the residence; since the workers have reduced the time spent on routine tasks and can assign this time to extra activities. Their work is automatically monitored, as well as the patients' activities. The stored information may be analysed with knowledge discovery techniques and may help to improve the quality of life for the patients and the efficiency of the centre [7]. The security of the centre has also been improved in two ways: the system monitors the patients and guarantees that each one of them is in the right place, and secondly, only authorised personnel can gain access to the residence protected areas. 
Table 2. Time (minutes) spent on indirect tasks

\begin{tabular}{cccccc}
\hline & Monitoring & Reports & Visits & Other & TOTAL \\
\hline Before & 167 & 48 & 73 & 82 & 370 \\
After & 105 & 40 & 45 & 60 & 250 \\
\hline
\end{tabular}

In the future, health care will require the use of new technologies that allow medical personnel to carry out their tasks more efficiently [4]. We have shown some potential of deliberative CBP-BDI agents in a distributed multi-agent system focused on health care. In addition, the use of RFID technology [10] on people provided a high level of interaction among users and patients through the system.

Acknowledgements. This work has been partially supported by the MCYT TIN200614630-C03-03.

\section{References}

1. Aamodt, A., Plaza, E.: Case-Based Reasoning: foundational Issues, Methodological Variations, and System Approaches. AICOM 7, 39-59 (1994)

2. Bajo, J., Corchado, J.M., Castillo, L.F.: Running agents in mobile devices. In: Sichman, J.S., Coelho, H., Rezende, S.O. (eds.) IBERAMIA 2006 and SBIA 2006. LNCS (LNAI), vol. 4140, pp. 58-67. Springer, Heidelberg (2006)

3. Bratman, M.E.: Intentions, Plans and Practical Reason. Harvard University Press, Cambridge, MA (1987)

4. Camarinha-Matos, L., Afsarmanesh, H.: Design of a virtual community infrastructure for elderly care. PRO-VE'02. Sesimbra, Portugal (2002)

5. Corchado, J.M., Laza, R.: Constructing Deliberative Agents with Case-based Reasoning Technology. International Journal of Intelligent Systems 18(12), 1227-1241 (2003)

6. Glez-Bedia, M., Corchado, J.M.: A planning strategy based on variational calculus for deliberative agents. Computing and Information Systems Journal 10(1), 2-14 (2002) ISBN: 1352-9404

7. Marrow, P.: Nature-Inspired computing technology and applications. BT Technology Journal, vol. 18(4), (2000)

8. Nealon, J., Moreno, A.: Applications of Software Agent Technology in the Health Care domain. Whitestein series in Software Agent Technologies (2003)

9. Pokahr, A., Braubach, L., Lamersdorf, W.: Jadex: Implementing a BDI-Infrastructure for JADE Agents. In Search of Innovation 3(3), 76-85 (2003)

10. Sokymat. Sokymat (2006), http://www.sokymat.com 